\title{
Isolated Systolic Hypertension and the Risk of Vascular Disease
}

\author{
Tim Nawrot, MSc*, Elly Den Hond, PhD, \\ Lutgarde Thijs, MSc, and Jan A. Staessen, MD, PhD
}

\begin{abstract}
Address
*Studiecoördinatiecentrum, Laboratorium Hypertensie, Department voor Moleculair en Cardiovasculair Onderzoek, Campus Gasthuisberg, Herestraat 49, B-3000 Leuven, Belgium.

E-mail: tim.nawrot@med.kuleuven.ac.be
\end{abstract}

Current Hypertension Reports 2003, 5:372-379

Current Science Inc. ISSN 1522-64I7

Copyright () 2003 by Current Science Inc.

Hypertension is present in $60 \%$ to $70 \%$ of the population over 60 years of age and may result in cardiovascular complications such as stroke, coronary heart disease, and heart failure. In this review, the role of arterial stiffness, endothelial function, atherosclerosis, and oxidative stress in the pathogenesis of hypertension is discussed extensively. Antihypertensive drug treatment may control high blood pressure and prevent complications. This review summarizes the results of several placebo-controlled and comparative clinical trials that have studied the efficacy of different classes of antihypertensive drugs.

\section{Introduction}

Essential hypertension refers to a lasting elevation of blood pressure with heterogeneous genetic and environmental causation. Its prevalence rises with age regardless of the type of blood pressure measurement and is dependent on the operational thresholds used for diagnosis. Aging is also associated with a steep increase in vascular diseases. This article reviews the risk between vascular diseases and isolated systolic hypertension from a clinical, epidemiologic, and pathophysiologic perspective.

\section{Epidemiology}

In cross-sectional and longitudinal population studies, systolic blood pressure (SBP) increases with age, while diastolic blood pressure (DBP) rises until 50 years of age and then levels off or even slightly decreases [1]. Consequently, with increasing age there is a shift from diastolic pressure to systolic pressure and then to pulse pressure as the predominant predictor of cardiovascular risk. In the Framingham Heart Study, DBP was the strongest predictor below age 50 years, all three blood pressure indexes were comparable predictors at ages 50 to 59, and from 60 years on, DBP was negatively related to risk of coronary events, so

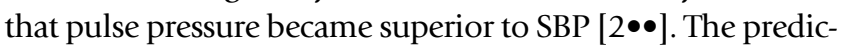
tive role of pulse pressure in the elderly is consistent in men and women, in treated and untreated hypertensive subjects [3•], and in patients with a history of myocardial infarction [4] or renal failure [5]. Furthermore, in older patients with isolated systolic hypertension, pulse pressure [6], and especially ambulatory pulse pressure [7], is a stronger predictor of cardiovascular risk than mean pressure.

According to guidelines proposed by the European Society of Hypertension (ESH)/European Society of Cardiology ESH/ESC [8], hypertension is defined as SBP of $140 \mathrm{~mm} \mathrm{Hg}$ or higher, DBP of $90 \mathrm{~mm} \mathrm{Hg}$ or higher, or taking antihypertensive drug treatment (Table 1). The prevalence of hypertension is about $25 \%$ in the general adult population and increases to $60 \%$ to $70 \%$ in the age group above 60 years [1]. In the general US population studied in the National Health and Nutrition Examination Survey III (NHANES III), men had a higher prevalence of hypertension than women up to 59 years, while the reverse was true from 60 years on [1].

Isolated systolic hypertension, defined as SBP of $140 \mathrm{~mm}$ $\mathrm{Hg}$ or higher and DBP below $90 \mathrm{~mm} \mathrm{Hg}$ (Table 1), is the predominant type of hypertension in the elderly. The NHANES III data demonstrated that below the age of 50 years, isolated diastolic hypertension (SBP below 140 and DBP $90 \mathrm{~mm} \mathrm{Hg}$ or more) was most common (46.9\%) among untreated hypertensives, while combined systolic/diastolic hypertension (SBP $140 \mathrm{~mm} \mathrm{Hg}$ or more and DBP $90 \mathrm{~mm} \mathrm{Hg}$ or more) was most common (45.1\%) among inadequately treated individuals. In both untreated and inadequately treated groups, isolated systolic hypertension became the primary hypertensive subtype for subjects after the age of 50 years.

Few studies provide information on the incidence of hypertension in the general population. In the Framingham Heart Study, 5.3\% of participants with optimal blood pressure, $17.6 \%$ with normal, and $37.3 \%$ with highnormal blood pressure below the age of 65 years developed hypertension over a period of 4 years $[9 \bullet]$.

\section{Pathophysiology}

Pulse pressure, the difference between SBP and DBP, is determined by the compliance of the arterial vascular bed and the 


\begin{tabular}{|c|c|c|c|}
\hline & Systolic blood press & & Diastolic blood pressure, $\mathrm{mm} \mathrm{Hg}$ \\
\hline \multicolumn{4}{|l|}{ Normotension } \\
\hline Optimal & $<120$ & and & $<80$ \\
\hline Normal & $120-129$ & or & $80-84$ \\
\hline High-normal & $130-139$ & or & $85-89$ \\
\hline \multicolumn{4}{|l|}{ Hypertension } \\
\hline Grade I (mild) & $140-159$ & or & $90-99$ \\
\hline Subgroup borderline & $\mid 40-149$ & or & $90-94$ \\
\hline Grade 2 (moderate) & $160-179$ & or & $100-109$ \\
\hline Grade 3 (severe) & $\geq 180$ & or & $\geq 110$ \\
\hline Isolated systolic hypertension & $\geq 140$ & or & $<90$ \\
\hline Subgroup borderline & $140-149$ & or & $<90$ \\
\hline
\end{tabular}

stroke volume, and to a lesser extent by the ejection rate of the left ventricle. Mean arterial pressure, a weighted average of SBP and DBP, is dependent on cardiac output and total peripheral resistance. Isolated systolic hypertension, by definition, is characterized by an increase in pulse pressure, but not necessarily by an increase in mean arterial pressure.

\section{Arterial stiffness as a determinant} of isolated systolic hypertension

Arterial stiffness is emerging as the most important determinant of primary isolated hypertension in our aging community. Arterial distensibility and pulse wave velocity reflect the elasticity of an artery. Aortic pulse wave velocity doubles while distensibility coefficients halve between 20 and 70 years of age, (Fig. $1 A$ and $B$ ). The arterial pressure wave consists of a forward component generated by the heart and reflected waves returning to the heart from peripheral sites [5]. In healthy young adults, the reflected waves coincide with diastole, raise diastolic pressure, and boost coronary perfusion. With arterial stiffening, the reflected waves move faster, reach the proximal aorta during systole, and cause an augmentation of late systolic pressures, whereas diastolic pressure decreases. The early return of the reflected pulse wave to the aorta during systole is the primary mechanism accounting for the rise in SBP and the decline in DBP that occurs with arterial stiffening. Lowered diastolic pressure may impair coronary blood flow and predispose to myocardial ischemia. The higher systolic pressure augments cardiac work and may lead to heart failure [5]. Histopathologic examination of the aorta of the elderly reveals thickening of the media due to the accumulation of collagenous fibers as well as calcium deposition [10]. A functional component may be superimposed on these structural changes. Indeed, the vasoconstrictor tone in the large arteries may well rise with age, when the number of $\beta_{2}$-adrenoreceptors that mediate vasodilatation decreases [11].

Endothelial function and arterial stiffness

Endothelial function estimated as flow-mediated vasodilatation apparently starts to deteriorate at about 50 years of age (Fig. 1C), ie, when pulse pressure begins to rise. Endothelial function is more per formant in premenopausal women than in men. This observation suggests that estrogens might enhance endothelial function. Indeed, estrogens activate endothelium-dependent vascular relaxation mechanisms, including the nitric oxide-cGMP and prostacyclin-cAMP pathways [12].

The endothelium generates a variety of biologic mediators that influence the tone and structure of the blood vessel and determine the susceptibility of the vessel wall to atherogenesis. One of these mediators is nitric oxide, which is synthesized from the amino acid L-arginine via the action of the enzyme nitric oxide synthase.

Endothelin-1 is an important vasoconstrictor released by the endothelium and may also increase arterial stiffness. Indeed, plasma endothelin-1 concentration shows a significant positive correlation with aortic stiffness in patients with coronary artery disease [13]. This observation illustrates that endothelial function affects the function of large arteries. Thus, large arteries are more than simply passive conduits.

\section{Atherosclerosis in isolated systolic hypertension}

Many of the histologic alterations that occur in the vessel wall with aging resemble those that come with artherosclerosis. Figure $1 D$ shows the increase in carotid intima media thickness, a sign of early atherosclerosis associated with advancing age. Nevertheless, the role of atherosclerosis in the pathogenesis of isolated systolic hypertension remains debatable. In autopsy specimens of human aorta, compliance decreases with age, but the relative contributions of aging and atherosclerosis remain unknown [14]. Moreover, clinical experience shows that many patients with severe generalized atherosclerosis maintain a normal central compliance. In contrast, in some populations with a low prevalence of atherosclerosis, systolic pressure does rise with age and isolated systolic hypertension is observed [15].

Hypertension: cause or effect of arterial stiffness?

Whether the acceleration of the aging process occurs through an increase in arterial stiffness or through hyper- 


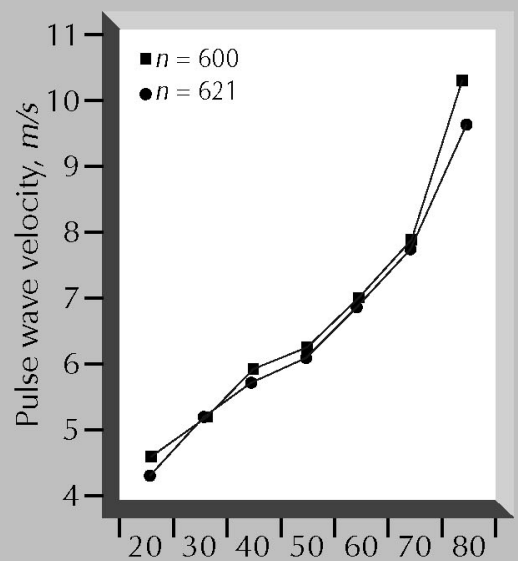

A

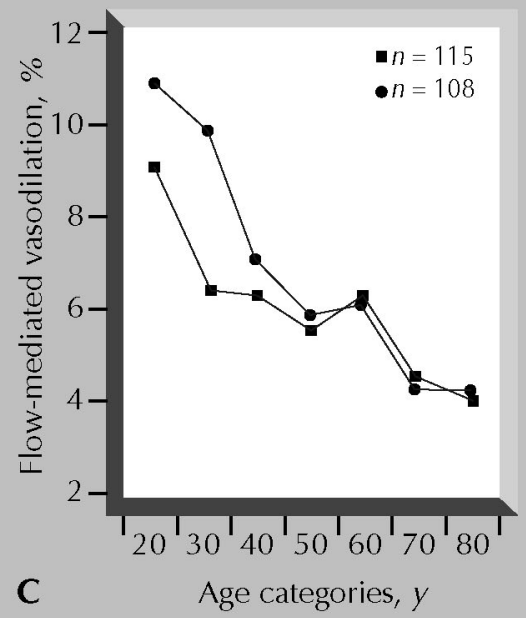

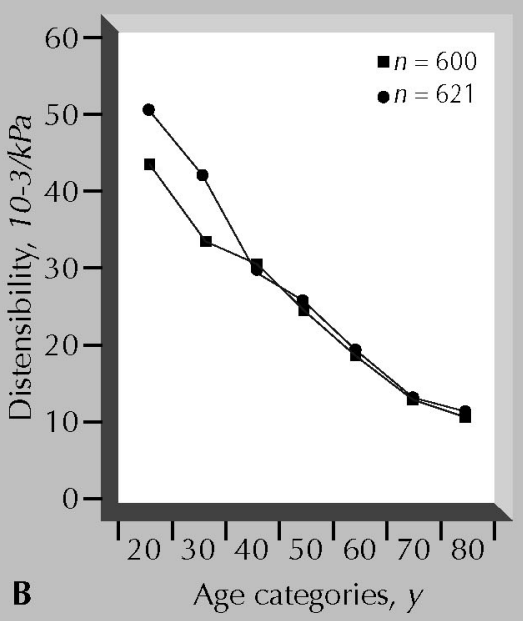

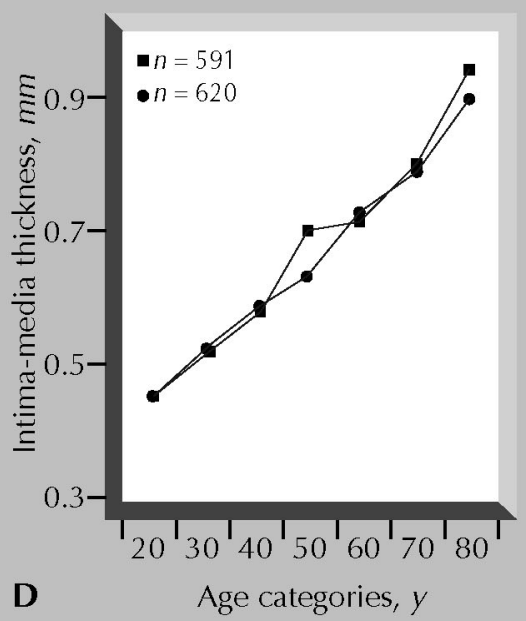

Figure 1. Wall characteristics by gender and age in a sample of the general population. Squares represent men and circles represent women. A, Pulse wave velocity; B, carotid artery distensibility; $\mathbf{C}$, endothelial function; D, intima-media thickness of the carotid artery. Data are mean for each age group. tension-associated changes in large artery structure has been debated. Liao et al. [16] evaluated the prospective relation between baseline arterial elasticity and the development of hypertension in a population sample. After 6 years of follow-up, one standard deviation decrease in arterial elasticity was associated with a $15 \%$ greater risk of hypertension, independent of established risk factors for hypertension and the level of baseline blood pressure. These results suggest that lower arterial elasticity is causally related to the development of systolic hypertension.

\section{Chronologic and biologic aging in arterial hemodynamics}

The age-related changes in cardiac function, circulatory hemodynamics, lipid metabolism, and detoxification of reactive oxygen species all contribute to morbidity and mortality in the elderly. Although age is an outstanding risk factor for high blood pressure, stroke, and coronary artery disease, the precise pathophysiologic mechanisms underlying these observations are presently unknown.

Telomeres, the end of chromosomes, prevent DNA "erosion" and are subject to attrition with each division of somatic cells. Recent experimental data support the concept that telomere length might serve as a biologic clock, not only at the cellular level but probably also at the systemic level $[17 \bullet \bullet, 18]$. Of interest is the fact that homocysteine, a known risk factor for atherosclerosis [19], enhances the rate of telomere attrition per replicative cycle in cultured human vascular endothelial cells [20]. To a large extent, this effect appears to be mediated by reactive oxygen species. Recently, it was shown that both pulse pressure and pulse wave velocity are inversely correlated with telomere length independent of chronologic age $[17 \bullet \bullet]$. These observations suggest that pulse pressure and pulse wave velocity might be markers of the biologic age of the vascular system.

\section{Established cardiovascular risk factors and arterial stiffness}

Obesity is associated with decreased aortic elasticity. The rise in blood pressure following gain in body weight is associated with profound changes in cardiovascular-renal physiology, including increased plasma volume, enhanced tubular sodium reabsorption, an initial decrease in total peripheral resistance, increased heart rate, activation of the sympathetic nervous system, and activation of the systemic renin-angiotensin-aldosterone axis [21]. Adipose tissue 
secretes the hormone leptin, which increases sympathetic activity [21].

Adults with familial hypercholesterolemia have a significantly lower aortic distensibility than control subjects [22]. The changes in vessel wall properties due to hypercholesterolemia may be due to 1) impaired responses to endothelial relaxing factors (such as nitric oxide), 2) an increased content of collagen and calcium subsequent to deposition of cholesterol in the vessel wall, and 3) a toxic effect of oxidized lipoproteins on the endothelium [23••].

Even in the young, cigarette smoking increases arterial stiffness, aortic blood pressure, and plasma von Willebrand factor (a marker of endothelial damage) [24,25]. More than 3800 chemicals present in tobacco smoke cause oxidative stress either directly or via biotransformation of these compounds. Smoking acutely increases sympathetic tone [26]. Furthermore, smoking decreases nitric oxide production, the primary vasodilator produced by endothelial cells [24].

Diabetes is associated with increased arterial stiffness and decreased endothelial function [27]. Glycation of proteins due to alterations in cellular metabolism remains a reasonable pathophysiologic link between hyperglycemia and the development of complications in diabetes. These chemically modified proteins may interact with cells in the vascular wall, inducing expression of cytokines and growth factors, which cause cross-linking of collagen molecules to each other [28], leading to enhanced loss of collagen elasticity with a subsequent reduction in arterial distensibility.

Homocysteine, a sulfur-containing amino acid that is formed in the metabolism of methionine to cysteine, behaves as an independent risk factor for cardiovascular disease. The two most important determinants of the plasma homocysteine concentration are folate status and renal function. From in vitro and animal models there is evidence that high homocysteine concentrations are associated with endothelial toxicity attributed to oxidative stress that results from auto-oxidation of homocysteine [29]. During this process hydrogen peroxide and superoxide are formed, which may increase low-density lipoprotein oxidation, and decrease the bioavailability of nitric oxide [29]. The decrease in nitric oxide leads to vasoconstriction. In hypertensive patients, plasma homocysteine is positively correlated with aortic stiffness as assessed by carotid-femoral pulse wave velocity, independent of sex, age, blood pressure, and renal function [30]. However, homocysteine-lowering therapy had no beneficial effect on carotid distensibility in patients with end-stage renal disease [31], or in healthy siblings of patients with premature atherosclerosis [32].

\section{Diagnosis}

According to the $2003 \mathrm{ESH} / \mathrm{ESC}$ guidelines for the management of arterial hypertension (World Health Organization [WHO]/International Society of Hypertension [ISH]) [8], the diagnosis of hypertension should be based on multiple conventional blood pressure measurements taken on separate occasions. However, ESH/ESC recommends that consideration should be given to blood pressure selfrecorded and/or ambulatory blood pressure monitoring before making therapeutic decisions [8]. A daytime ambulatory or self-recorded blood pressure of 135/85 mm Hg may be regarded as hypertensive [8].

Ambulatory and self-recorded blood pressure monitoring significantly increase the prognostic accuracy of conventional blood pressure readings, both in hypertensive patients [33] and in the general population [34]. The greater number of measurements, the absence of digit preference and observer bias, and the minimization of the white coat effect contribute to the greater reproducibility and consequently to the predictive superiority of ambulatory and self-recorded blood pressures over conventional pressures. In addition, a blunted fall in nocturnal blood pressure is a harbinger of an unfavorable outcome [35].

In the clinical setting, the main use of ambulatory blood pressure monitoring is to identify patients with white coat hypertension in whom antihypertensive drug treatment may be avoided. White coat hypertension is usually defined as an elevation of the clinic blood pressure in the presence of a normal daytime ambulatory blood pressure [36]. In older patients with isolated systolic hypertension, clinic and daytime ambulatory blood pressure measurements may differ largely. Event-based studies [37] have clearly demonstrated that the risk of cardiovascular disease is lower in patients with white coat hypertension than in those with higher ambulatory blood pressure even after controlling for concomitant risk factors. When daytime ambulatory blood pressure was below $130 \mathrm{~mm} \mathrm{Hg}$ systolic and $80 \mathrm{~mm} \mathrm{Hg}$ diastolic, the incidence of cardiovascular disorders did not differ between normotensive subjects and those with white coat hypertension [36]. In the Syst-Eur trial, patients with isolated systolic hypertension (conventional SBP $160 \mathrm{~mm} \mathrm{Hg}$ or more) were classified according to daytime SBP into one of three subgroups: nonsustained hypertension (less than $140 \mathrm{~mm} \mathrm{Hg}$ ), mild sustained hypertension (140 to $159 \mathrm{~mm} \mathrm{Hg}$ ), and moderate sustained hypertension (160 mm Hg or more). Patients with nonsustained hypertension had smaller electrocardiogram (ECG) voltages and a lower incidence of stroke and of cardiovascular complications than patients with moderate sustained hypertension [38]. The counterpart of white coat hypertension is masked hypertension, a disorder characterized by a normal conventional blood pressure confirmed on repeated clinic visits, but an elevated daytime ambulatory blood pressure. Preliminary observations suggest that this condition is associated with more targetorgan damage than observed in subjects with sustained normotension [39].

Conventional blood pressure measurement remains the standard for evaluating blood pressure. In patients with raised clinic blood pressure and either target-organ damage or a high cardiovascular risk profile, treatment can be started 
without confirmation of the elevated clinic readings by automated measurement techniques. When, however, a raised blood pressure is the only detectable abnormality or when patients with a normal clinic blood pressure show unexplained target-organ damage, self-measurement, ambulatory monitoring, or both must be used to exclude white coat hypertension or masked hypertension, respectively. In the absence of other cardiovascular risk factors and signs of target organ damage, therapy of patients with white coat hypertension may be limited to lifestyle measures and annual followup of the ambulatory blood pressure [40].

\section{Complications}

Increased blood pressure may result in cardiac, cerebral, retinal, or renal complications. Within the scope of this article, only cardiovascular complications will be discussed.

The heart responds to hypertension with compensatory growth of the myocardium, thus resulting in left ventricular hypertrophy and, if left untreated, followed by dilatation and heart failure. Besides affecting the myocardium directly, hypertension causes marked adaptive changes in the circulation and is a major risk factor for coronary heart disease and stroke. In middle-aged Europeans and Americans, the main complication of hypertension is coronary heart disease, whereas in Asians and older people it is stroke [41]. Blacks tend to have higher levels of blood pressure and hypertension-related mortality rates than do nonblacks [42].

In a collaborative project [43] using data from nine prospective studies including 418,343 individuals between 25 and 84 years of age, mainly men (96\%), an increase of 5 $\mathrm{mm} \mathrm{Hg}$ diastolic, accompanied by a $9 \mathrm{~mm} \mathrm{Hg}$ higher systolic pressure, conferred a 35\% to $40 \%$ higher risk of stroke and a $20 \%$ to $25 \%$ higher risk of coronary disease. The Framingham Heart Study showed that after a 30-year follow-up, hypertensives compared with normotensives had a sixfold higher risk for heart failure and a twofold higher risk for intermittent claudication [44].

Randomized clinical trials provide data on the reversibility of the risk associated with hypertension, although usually follow-up is no longer than 3 to 5 years. In eight trials, 15,693 patients with isolated systolic hypertension were followed-up for 3.8 years (median). After correction for regression dilution bias, sex, age, and $\mathrm{DBP}$, the relative hazard rates associated with a $10 \mathrm{~mm} \mathrm{Hg}$ higher initial SBP were $1.26(P<0.001)$ for total mortality, $1.22(P=0.02)$ for stroke, but only $1.07(P=0.37)$ for coronary events. Independent of SBP, DBP was inversely correlated with total mortality, highlighting the role of pulse pressure as risk factor.

\section{Outcome Trials \\ Placebo-controlled trials}

The ultimate goal of treating patients with isolated systolic hypertension is not to reduce blood pressure, but to prevent the cardiovascular and renal complications of an elevated SBP so that survival is prolonged and the quality of life is improved. Since 1991, three outcome trials have been published that addressed the question of whether in the elderly the cardiovascular risk conferred by isolated systolic hypertension is reversible by antihypertensive drug treatment. In all trials and subgroups combined [3•], among 7757 control patients, 734 deaths and 835 major cardiovascular complications occurred; in 7936 patients allocated active treatment, these numbers were 656 and 647, respectively. Median follow-up was 3.8 years. Overall, active treatment reduced total mortality by $13 \%$, cardiovascular mortality by $18 \%$, all cardiovascular complications by $26 \%$, stroke by $30 \%$, and coronary events by $23 \%$ [3•].

In the Systolic Hypertension in Europe (Syst-Eur) trial patients (60 years or older) with isolated systolic hypertension were randomized to active treatment $(n=2398)$, ie, nitrendipine, with the possible addition of enalapril and hydrochlorothiazide, or to matching placebos $(n=2297)$ [45]. The between-group difference in blood pressure amounted to $10.1 / 4.5 \mathrm{~mm} \mathrm{Hg}(P<0.001)$. Active treatment reduced the incidence of fatal and nonfatal stroke by $42 \%$ and that of major cardiovascular complications by $30 \%$. Cardiovascular mortality tended to be lower on active treatment $(-26 \%, P=0.08)$, but all-cause mortality was not significantly influenced $(-13 \%, P=0.28)[45,46]$. Furthermore, active treatment reduced the incidence of mild renal dysfunction (serum creatinine $176.8 \mu \mathrm{mol} / \mathrm{L}$ or more) by $64 \%$ (13 vs five cases, $P=0.04$ ) [47]. The relative benefits of active treatment were evenly distributed across the two sexes and across patients with and without a history of cardiovascular complications [48]. Further analysis also suggested similar benefit in patients who remained on nitrendipine monotherapy [49].

At randomization into the Syst-Eur trial, 492 patients $(10.5 \%)$ had diabetes mellitus. In these patients, with adjustments for possible confounders, active treatment reduced all-cause mortality by 55\%, cardiovascular mortality by $76 \%$, all cardiovascular endpoints by $69 \%$, fatal and nonfatal strokes by $73 \%$, and all cardiac endpoints by $63 \%$. The reductions in total mortality $(P=0.04)$, cardiovascular mortality $(P=0.02)$, and all cardiovascular endpoints $(P=0.01)$ were significantly larger in diabetic than in nondiabetic patients (Fig. 2) [50]. Furthermore, active treatment reduced the risk of proteinuria more $(P=0.04)$ in diabetic than in nondiabetic patients (71\% vs $20 \%)$.

The Syst-Eur Vascular Dementia Substudy [51] investigated whether antihypertensive treatment could reduce the incidence of dementia. In total, 2418 patients were enrolled. After a median follow-up of 2 years in the double-blind trial, active treatment reduced the incidence of dementia by $50 \%(P=0.05)$ from 7.7 to 3.8 cases per 1000 patient-years (11 vs 21 patients). Active treatment prevented mainly Alzheimer's dementia (eight vs 15 cases). These results were confirmed in the open-label follow-up study [52]. During extended follow-up, the number of incident cases of dementia increased from 32 to 64 . Long-term active treatment $(n=1485)$ compared with control $(n=$ 


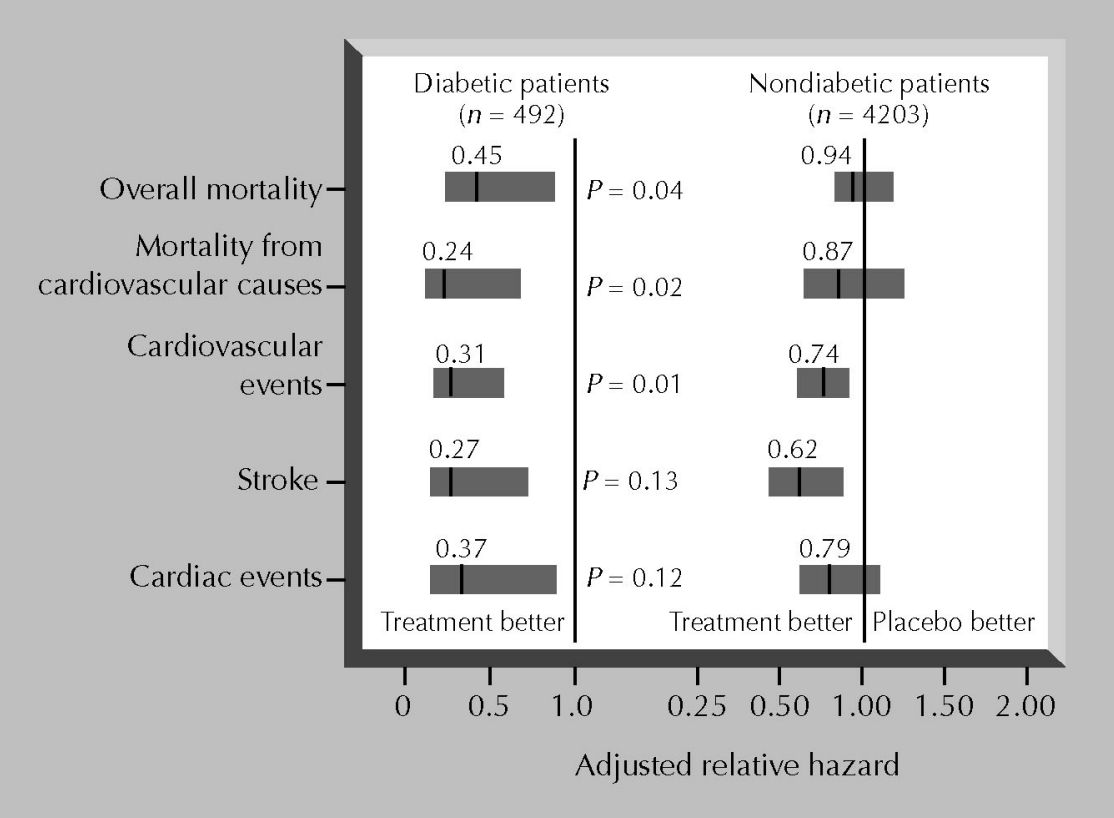

Figure 2. Adjusted relative hazard rates associated with active treatment as compared with placebo in diabetic and nondiabetic patients participating in the Syst-Eur trial. The relative hazard rates were adjusted for sex, age, previous cardiovascular complications, systolic blood pressure at enrollment, smoking status, and residence in eastern or western Europe. The $P$ values are for the interaction between treatment and diabetes and indicate whether the treatment effect was significantly associated with the presence or absence of diabetes at enrollment. Cardiovascular events, stroke, and cardiac events included fatal and nonfatal events. The bars indicate the $95 \%$ confidence intervals. The numbers above the bars indicate the benefit of the active treatment compared with placebo. (Adapted from Tuomilehto et al. [50].)

1417) reduced the incidence of dementia by 55\%, from 7.4 to 3.3 cases per 1000 patient-years (43 vs 21 patients) [52]. Several reports suggest that calcium channel blockers, which cross the blood-brain barrier and bind to brain receptors located in areas affected by Alzheimer's disease, may confer specific neuroprotection [53]. However, the concept that antihypertensive therapy with long-acting dihydropyridines might protect against dementia needs further testing in controlled clinical trials.

The double-blind Syst-Eur trial $(P=0.009)$ [48] showed an interaction between treatment allocation and age. Randomization to active treatment was associated with a decreased relative risk of death in subjects aged 60 to 69 years (59\%) and 70 to 79 years (58\%), but with a slightly increased risk of death in patients aged 80 years or more $(11 \%)$. Similarly, a meta-analysis of the results of controlled trials in hypertensive patients over the age of 80 years showed a nonsignificant $6 \%$ excess of death from all causes [54]. In view of the remaining uncertainty, the Hypertension in the Very Elderly Trial (HYVET) is currently investigating the potential benefit of antihypertensive drug treatment on stroke and other cardiovascular endpoints in the very elderly [55]. This trial will include a subgroup of patients with isolated systolic hypertension.

All available outcome trials in isolated systolic hypertension recruited patients with an SBP of $160 \mathrm{~mm} \mathrm{Hg}$ or more. Currently no data are available showing that antihypertensive drug treatment reduces the risk of cardiovascular events in patients with borderline isolated systolic hypertension (SBP between 140 and $159 \mathrm{~mm} \mathrm{Hg}$ and DBP less than 90 $\mathrm{mm} \mathrm{Hg}$ ) who have no previous cardiovascular complications and who are not exposed to other risk factors. Because the prevalence of this condition may be estimated to be around $20 \%$ [56] and because borderline isolated systolic hypertension is probably associated with an increased cardiovascular risk [57], outcome trials in patients with borderline isolated systolic hypertension are urgently needed.

\section{Comparative trials}

Sixteen recent outcome trials compared old classes of antihypertensive drugs, such as diuretics and $\beta$-blockers, with new agents such as calcium channel blockers, angiotensinconverting enzyme (ACE) inhibitors, angiotensin type 1

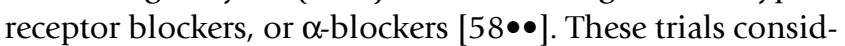
ered mainly patients with combined systolic and diastolic hypertension. Some trials [59-62] included subgroups of patients with isolated systolic hypertension. However, none of the trials provided specific estimates of the treatment differences in patients with isolated systolic hypertension. In all 135,842 patients with systolic and diastolic hypertension combined, old and new drugs provided similar protection against total and cardiovascular mortality and fatal and nonfatal myocardial infarction. Calcium channel blockers, including $(+8 \%, P=0.07)$ or excluding $(+10 \%, P=0.02)$ verapamil, as well as angiotensin type 1 receptor blockers $(+24 \%, P=0.0002)$ resulted in better stroke prevention than did the old drugs, whereas the opposite trend was observed for ACE inhibitors $(-10 \%, P=$ $0.03)$. The risk of heart failure was higher $(P<0.0001)$ on calcium channel blockers (+33\%) and $\beta$-blockers $(+102 \%)$

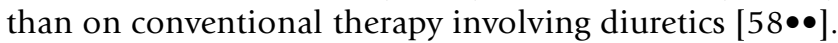
These results should be interpreted with caution. Indeed, more detailed analyses demonstrated that most differences in specific cardiovascular endpoints were due to design

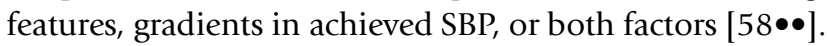

\section{Conclusions}

Since age and blood pressure are continuous variables with no discernible transition points where there is a sudden 
increase in morbidity and mortality, arbitrary definitions must be used for classification. Unlike DBP, SBP increases progressively with age. The characteristic changes of SBP and DBP with age lead to an increase in pulse pressure, which has emerged as a new and potentially independent risk factor reflecting the biologic aging of the vascular system.

Arterial stiffness is increasingly recognized as an important factor underlying cardiovascular complications in the elderly. Population and clinical studies suggest that SBP should be the primary target of antihypertensive therapy, although consideration of SBP and DBP together improves risk prediction.

\section{References and Recommended Reading}

Papers of particular interest, published recently,

have been highlighted as:

- Of importance

$\bullet \quad$ Of major importance

1. Staessen J, Amery A, Fagard R: Editorial review. Isolated systolic hypertension in the elderly. J Hypertens 1990, 8:393-405.

2.•• Franklin SS, Larson MG, Khan SA, et al.: Does the relation of blood pressure to coronary heart disease change with aging? Circulation 2001, 103:1245-1249.

This epidemiologic study shows that with increasing age there is a gradual shift from diastolic to systolic and then to pulse pressure as predictor of coronary heart disease risk. The strength of the study is the large number of subjects ( $>6500)$ in a wide age range (20-79 years) with a 20-year follow-up.

3. Staessen JA, Gasowski J, Wang JG, et al:: Risks of untreated and treated isolated systolic hypertension in the elderly: meta-analysis of outcome trials. Lancet 2000, 355:865-872.

This meta-analysis estimates the risk for total mortality and cardiovascular events in patients with isolated systolic hypertension. The paper confirms risk reduction scores found in epidemiologic studies and also gives subgroup analyses by sex, age, previous complications, smoking, SBP, and pulse pressure.

4. Mitchel GF, Moyé LA, Braunwald E, et al., for the SAVE Investigators: Sphygmomanometrically determined pulse pressure is a powerful independent predictor of recurrent events after myocardial infarction in patients with impaired left ventricular function. Circulation 1997, 96:4254-4260.

5. Safar ME, Blacher J, Pannier B, et al.: Central pulse pressure and mortality in end-stage renal disease. Hypertension 2002, 39:735-738.

6. Blacher J, Staessen JA, Girerd X, et al.: Pulse pressure not mean pressure determines cardiovascular risk in older hypertensive patients. Arch Intern Med 2000, 160:1085-1089.

7. Staessen JA, Thijs L, O'Brien ET, et al., for the Systolic Hypertension in Europe (Syst-Eur) Trial Investigators: Ambulatory pulse pressure as predictor of outcome in older patients with systolic hypertension. Am J Hypertens 2002, 15(part 1):835-843.

8. Guidelines Committee: ESH hypertension guidelines. J Hypertens 2003, In press.

9. Vasan RS, Larson MG, Leip EP, et al.: Assessment of frequency to progression to hypertension in non-hypertensive participants in the Framingham Heart Study: a cohort study. Lancet 2001, 358:1682-1686.

This study showed that approximately $40 \%$ of the persons with high-normal blood pressure below the age of 65 years will develop hypertension over a period of 4 years.

10. Asmar R, Safavian A, Tual JL, Safar ME: Arterial and cardiac changes in hypertension in the elderly. Blood Press Suppl 1995, 3:31-37.
11. Schocken DD, Roth GS: Reduced beta-adrenergic receptor concentrations in ageing man. Nature 1977, 267:856-8.

12. Thompson J, Khalil RA: Gender differences in the regulation of vascular tone. Clin Exp Pharmacol Physiol 2003, 30:1-15.

13. Heintz B, Dorr R, Gillessen T, et al.: Do arterial endothelin 1 levels affect local arterial stiffness? Am Heart J 1993, 126:987-989.

14. Hallock P, Benson IC: Studies on the elastic properties of human isolated aorta. J Clin Invest 1937, 16:595-602.

15. M'Buyamba-Kabangu JR, Fagard R, et al.: Blood pressure and urinary cations in urban Bantu of Zaire. Am J Epidemiol 1986, 124:957-968.

16. Liao D, Arnett DK, Tyroler HA, et al.: Arterial stiffness and the development of hypertension. The ARIC study. Hypertension 1999, 34:201-206.

17. $\bullet$ Benetos A, Okuda K, Lajemi M, et al.: Telomere length as an indicator of biological aging: the gender effect and relation with pulse pressure and pulse wave velocity. Hypertension 2001, 37:381-385.

This study shows that telomeres, a marker of biologic age, are associated with pulse pressure and pulse wave velocity in men independent from chronologic age. It suggests that pulse pressure and pulse wave velocity are markers of the biologic age of the vascular.

18. Aviv A, Aviv H: Reflections on telomeres, growth, aging, and essential hypertension. Hypertension 1997, 29:1067-1072.

19. Stein JH, McBride PE: Hyperhomocysteinemia and atherosclerotic vascular disease. Pathophysiology, screening and treatment. Arch Intern Med 1998, 158:1301-1306.

20. Xu D, Neville R, Finkel T: Homocysteine accelerates endothelial cell senescence. FEBS Lett 2000, 470:20-24.

21. Mark AL, Correia M, Morgan DA, et al.: State-of-the-art-lecture: Obesity-induced hypertension: new concepts from the emerging biology of obesity. Hypertension 1999, 33:5375-41.

22. Lehmann ED, Hopkins KD, Gosling RG: Aortic compliance measurements using Doppler ultrasound: in vivo biochemical correlates. Ultrasound Med Biol 1993, 19:683-710.

23.• Mertens A, Holvoet P: Oxidized LDL and HDL: antagonists in atherothrombosis. FASEB J 2001, 15:2073-2084.

This review focus on the presence of different forms of oxidatively modified low-density lipoprotein in the circulation and their relation to cardiovascular disease.

24. Mahmud A, Feely J: Effect of smoking on arterial stiffness and pulse pressure amplification. Hypertension 2003, 41:183-187.

25. Prisco D, Fedi S, Brunelli T, et al.: The influence of smoking on von Willebrand factor is already manifest in healthy adolescent females: the Floren-teen (Florence Teenager) Study. Int J Clin Lab Res 1999, 29:150-154.

26. Winniford MD: Smoking and cardiovascular function. J Hypertens 1990, 8(Suppl):S17-S23.

27. Wiltshire EJ, Gent R, Hirte C, et al.: Endothelial dysfunction relates to folate status in children and adolescents with type 1 diabetes. Diabetes 2002, 51:2282-2286.

28. Larsen ML, Horder M, Mogensen EF: Effect of long-term monitoring of glycosylated hemoglobin levels in insulin-dependent diabetes mellitus. N Engl J Med 1990, 323:1021-1025.

29. Welch GN, Loscalzo J: Homocysteine and atherothrombosis. N Engl J Med 1998, 338:1042-1050.

30. Bortolotto LA, Safar ME, Billaud E, et al.: Plasma homocysteine, aortic stiffness, and renal function in hypertensive patients. Hypertension 1999, 34:837-842.

31. van Guldener C, Lambert J, ter Wee PM, et al.: Carotid artery stiffness in patients with end-stage renal disease: no effect of long-term homocysteine-lowering therapy. Clin Nephrol 2000, 53:33-41.

32. van Dijk RA, Rauwerda JA, Steyn M, et al.: Long-term homocysteine-lowering treatment with folic acid plus pyridoxine is associated with decreased blood pressure but not with improved brachial artery endothelium-dependent vasodilation or carotid artery stiffness: a 2-year, randomized, placebo-controlled trial. Arterioscler Thromb Vasc Biol 2001, 21:2072-2079. 
33. Staessen JA, Thijs L, Fagard R, et al., for the Systolic Hypertension in Europe Trial Investigators: Predicting cardiovascular risk using conventional and ambulatory blood pressure in older patients with systolic hypertension. JAMA 1999, 282:539-546.

34. Ohkubo T, Imai Y, Tsuji I, et al:: Home blood pressure measurement has a stronger predictive power for mortality than does screening blood pressure measurement: a population-based observation in Ohasama, Japan. J Hypertens 1998, 16:971-975.

35. Kario K, Pickering TG, Matsuo T, et al.: Stroke prognosis and abnormal nocturnal blood pressure falls in older hypertensives. Hypertension 2001, 38:852-857.

36. Verdecchia P, Staessen J, White WB, et al.: Properly defining white coat hypertension. Eur Heart J 2002, 23:106-109.

37. Verdecchia P, Schillaci G, Borgioni C, et al.: Ambulatory pulse pressure. A potent predictor of total cardiovascular risk in hypertension. Hypertension 1998, 32:983-988.

38. Fagard RH, Staessen JA, Thijs L, et al., for the Systolic Hypertension in Europe (Syst-Eur) Trial Investigators: Response to antihypertensive therapy in older patients with sustained and nonsustained systolic hypertension. Circulation 2000, 102:1139-1144.

39. Sega R, Trocino G, Lanzarotti A: Alterations of cardiac structure in patients with isolated office, ambulatory, or home hypertension: data from the general population (Pressione Arteriose Monitorate E Loro Associazioni [PAMELA] Study). Circulation 2002, 104:1385-1392.

40. Staessen JA, Byttebier G, Buntinx F, et al., for the Ambulatory Blood Pressure Monitoring and Treatment of Hypertension Investigators: Antihypertensive treatment based on conventional or ambulatory blood pressure measurement. A randomized controlled trial. JAMA 1997, 278:1065-1072.

41. Wu K, Xie L, Chen D, Chen J: The natural history of borderline hypertension in a Chinese population. J Hum Hypertens 1997, 11:95-100.

42. Gillum RF: The epidemiology of cardiovascular disease in black Americans. N Engl J Med 1996, 335:1597-598.

43. MacMahon S, Peto R, Cutler J, et al.: Blood pressure, stroke, and coronary heart disease. Part 1, prolonged differences in blood pressure : prospective observational studies corrected for the regression dilution bias. Lancet 1990, 335:765-774.

44. Stokes J, Kannel WB, Wolf PA, et al.: Blood pressure as a risk factor for cardiovascular disease. The Framingham Study - 30 years of follow-up. Hypertension 1989, 13(Suppl I):113-118.

45. Staessen JA, Thijs L, Birkenhäger $\mathrm{WH}$, et al., on behalf of the Syst-Eur Investigators: Update on the Systolic Hypertension in Europe (Syst-Eur) Trial. Hypertension 1999, 33:1476-1477.

46. Staessen JA, Fagard R, Thijs L, et al., for the Systolic Hypertension in Europe (Syst-Eur) Trial Investigators: Randomised double-blind comparison of placebo and active treatment for older patients with isolated systolic hypertension. Lancet 1997, 350:757-764 [correction published in Lancet 1997, 350:1636].

47. Voyaki SM, Staessen JA, Thijs L, et al., for the Systolic Hypertension in Europe (Syst-Eur) Trial Investigators: Follow-up of renal function in treated and untreated older patients with isolated systolic hypertension. J Hypertens 2001, 19:511-19.

48. Staessen JA, Fagard R, Thijs L, et al., for the Systolic Hypertension in Europe (Syst-Eur) Trial Investigators: Subgroup and per-protocol analysis of the randomized European trial on isolated systolic hypertension in the elderly. Arch Intern Med 1998, 158:1681-1691.
49. Staessen JA, Thijs L, Fagard RH, et al., for the Systolic Hypertension in Europe (Syst-Eur) Trial Investigators: Calcium channel blockade and cardiovascular prognosis in the European trial on isolated systolic hypertension. Hypertension 1998, 32:410-416.

50. Tuomilehto J, Rastenyte $\mathrm{D}$, Birkenhäger $\mathrm{WH}$, et al., for the Systolic Hypertension in Europe (Syst-Eur) Trial Investigators: Effects of calcium-channel blockade in older patients with diabetes and systolic hypertension. $N$ Engl J Med 1999, 340:677-684.

51. Forette F, Seux ML, Staessen JA, et al., on behalf of the SystEur Investigators: Prevention of dementia in randomised double-blind placebo-controlled Systolic Hypertension in Europe (Syst-Eur) trial. Lancet 1998, 352:1347-1351.

52. Forette F, Seux ML, Staessen JA, et al., for the Syst-Eur Investigators: The prevention of dementia with antihypertensive treatment. New evidence from the Systolic Hypertension in Europe (Syst-Eur) Study. Arch Intern Med 2002, 162:2046-2052.

53. Parnetti L, Senin U, Mecocci P: Cognitive enhancement therapy for Alzheimer's disease. The way forward. Drugs 1997, 53:752-768.

54. Gueyffier F, Bulpitt C, Boissel JP, et al., for the INDANA Group: Antihypertensive drugs in very old people: a subgroup meta-analysis of randomised controlled trials. Lancet 1999, 353:793-796

55. Beckett NS, Connor M, Sadler JD, et al., on behalf of the HYVET Investigators: Orthostatic fall in blood pressure in the very elderly hypertensive: results from the Hypertension in the Very Elderly Trial (HYVET) - pilot. J Hum Hypertens 1999, 13:839-840.

56. Staessen JA, Wang JG, Brand E, et al.: Effects of three candidate genes on prevalence and incidence of hypertension in a Caucasian population. J Hypertens 2001, 19:1349-1358.

57. Himmelmann A, Hedner T, Hansson L, et al.: Isolated systolic hypertension : an important cardiovascular risk factor. Blood Press 1998, 7:197-207.

$58 . \bullet$ Staessen JA, Wang JG, Thijs L: Cardiovascular prevention and blood pressure reduction: a meta-analysis. Lancet 2001, 358:1305-1315 [correction published in Lancet 2002, 359:360].

This meta-regression analysis shows that the benefits of antihypertensive therapy largely depend on blood pressure lowering. This paper could not confirm the hypothesis that newer antihypertensive drugs influence cardiovascular prognosis over and beyond their blood pressure-lowering effects.

59. Hansson L, Lindholm LH, Ekbom T, et al., for the STOP-Hypertension-2 Study Group: Randomised trial of old and new antihypertensive drugs in elderly patients: cardiovascular mortality and morbidity in the Swedish Trial in Old Patients with Hypertension-2 study. Lancet 1999, 354:1751-1756.

60. UK Prospective Diabetes Study Group: Efficacy of atenolol and captopril in reducing risk of macrovascular and microvascular complications in type 2 diabetes: UKPDS 39. Br Med J 2000, 317:713-720.

61. Dahlöf B, Devereux RB, Kjeldsen SE, et al., for The LIFE Study Group: Cardiovascular morbidity and mortality in the Losartan Intervention For Endpoint reduction in hypertension study (LIFE): a randomised trial against atenolol. Lancet 2002, 359:995-1003.

62. Wing LMH, Reid CM, Ryan P, et al., for the Second Australian National Blood Pressure Study Group: A comparison of outcomes with angiotensin-converting-enzyme inhibitors and diuretics for hypertension in the elderly. $N$ Engl J Med 2003, 348:583-592. 